\title{
Enzalutamide for the treatment of prostate cancer
}

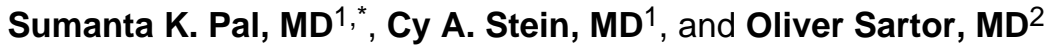 \\ ${ }^{1}$ Department of Medical Oncology \& Experimental Therapeutics, City of Hope Comprehensive \\ Cancer Center, Duarte, CA \\ ${ }^{2}$ Department of Urology, Tulane University School of Medicine, New Orleans, LA
}

\begin{abstract}
Introduction-The FDA approval of docetaxel for metastatic castration-resistant prostate cancer (mCRPC) in 2005 marked a major milestone - as it was the first approved agent for this disease that demonstrated a survival advantage in phase III assessment in this disease. Since 2009, several other agents have been FDA approved, including sipuleucel-T, abiraterone, cabazitaxel and enzalutamide. Enzalutamide, a potent antiandrogen that blocks nuclear translocation of the androgen receptor (AR) is the most recently approved of these agents.
\end{abstract}

Areas Covered-The clinical development of enzalutamide is discussed, with attention given as to how this agent will most appropriately be used among a growing list of agents for mCRPC. A MEDLINE search was conducted to identify all relevant published datasets pertaining to the drug. In addition, relevant ASCO and ESMO abstracts were searched.

Expert Opinion-The current role and sequencing of enzalutamide may change drastically based on studies such as PREVAIL (a phase III pre-chemotherapy assessment of enzalutamide) and planned studies to assess relevant combinations (i.e., enzalutamide with abiraterone). Outside of clinical efficacy, issues such as drug cost may ultimately dictate our utilization of agents such as enzalutamide for mCPRC. Although the development of biomarkers to guide therapy for mCRPC is ideal, there are inherent challenges in establishing biomarker-driven treatment.

\section{Keywords}

Enzalutamide; MDV3100; prostate cancer; phase III; castration resistant; hormone resistant; AFFIRM; PREVAIL

\subsection{Introduction}

\begin{abstract}
Although metastatic castration-resistant prostate cancer (mCRPC) remains incurable, there have been several critical advances in recent years. Four systemic therapies for mCRPC that prolong survival have been FDA approved within the past 3 years (Figure 1). On April 29, 2010, the FDA approved sipuleucel-T, an autologous dendritic cell vaccine that demonstrated a survival advantage relative to placebo in the phase III IMPACT trial. ${ }^{1-2}$ Whereas the IMPACT trial largely assessed patients that were chemotherapy-naïve, the phase III TROPIC trial compared cabazitaxel and mitoxantrone in patients that had failed docetaxel-based regimens. ${ }^{3}$ A survival advantage in this study led to the FDA approval of cabazitaxel on June $17,2010 .{ }^{4}$ The phase III COU-AA-301 study also included a docetaxel-
\end{abstract}

\footnotetext{
"Corresponding Author: Department of Medical Oncology \& Experimental Therapeutics City of Hope Comprehensive Cancer Center 1500 East Duarte Road Duarte, CA 91010 Office: (626) 256-4673 Fax: (626) 301-8233 spal@ coh.org.

Declaration of interest: Sumanta K. Pal and Cy A. Stein have received honoraria from Medivation. Oliver A. Sartor is a consultant for Astellas Pharmaceuticals.
} 
refractory population, and compared the CYP17 hydroxylase/lyase inhibitor abiraterone to placebo. ${ }^{5}$ Both this trial and the subsequently reported COU-AA-302 trial (which studied a chemotherapy-naïve population) met their primary endpoints, leading to the approval of abiraterone for docetaxel-refractory and docetaxel-naïve patients on April 28, 2011 and December 10, 2012, respectively. ${ }^{6-7}$

The most recent agent to be evaluated in the mCRPC disease space is enzalutamide, a novel antiandrogen that inhibits nuclear translocation of the androgen receptor (AR). Enzalutamide also has a higher binding affinity for AR as compared to standard antiandrogens (i.e., bicalutamide). In the phase III AFFIRM trial, enzalutamide was compared to placebo in docetaxel-refractory patients. ${ }^{8}$ As discussed later, the study met its primary endpoint, leading to FDA approval of the drug on August 31, 2012. ${ }^{9}$ With four agents now available that prolong survival in mCRPC, the question of sequencing and combinations now emerges. In addition, it is unclear which patients, if any, will benefit from one drug as opposed to another. For example, in the docetaxel-naïve patient, should abiraterone be sequenced prior to sipuleucel-T, or vice versa? In the docetaxel-refractory space, there is now a choice between cabazitaxel and enzalutamide. This manuscript will provide a detailed overview of the clinical development of enzalutamide, with commentary focused on the application of this drug in an increasingly crowded therapeutic space.

\subsection{Preclinical Development}

Several mechanisms for hormonal resistance have been postulated. Mutations in the androgen receptor (AR) may occur in patients with mCRPC, and several mutations have been localized to the ligand-binding domain (LBD) of the AR. ${ }^{10-12}$ Alternatively, bypass mechanisms (i.e., signaling via various transmembrane receptors) may circumvent increased prostate cancer mitogenesis via the AR. ${ }^{13}$ Elegant preclinical studies have also demonstrated AR overexpression as a mechanism of hormone resistance, contingent upon the presence of a functional LBD (Splice variants lacking a functional LBD are discussed later in this review). ${ }^{14-15}$ Although levels of androgen are physiologically low in the setting of the castration-resistant disease, the higher levels of AR may be compensatory and permit persistent AR-mediated signaling. Thus, agents have been developed that either (1) more potently inhibit androgen synthesis (i.e., abiraterone), or (2) more potently antagonize AR.

Enzalutamide falls into the latter category (Table 1). The agent was first identified from a library of compounds derived from the chemical scaffold of RU59063, an antiandrogen with modest affinity for the AR. ${ }^{16}$ Enzalutamide and a second antiandrogen, RD162, were selected for further development based on their binding properties to the AR and their pharmacokinetic characteristics. Both agents demonstrated a 5-8 fold higher affinity for the AR relative to bicalutamide, with a binding constant for AR in the low nanomolar range, and both led to decreased expression of products of AR-regulated genes, such as PSA and TMPRSS2. To evaluate these drugs in vivo, mice were castrated and then implanted with LNCaP/AR tumors, which over express wild-type AR. In this model, treatment with RD162 or enzalutamide resulted in a marked reduction in tumor volume. In a direct comparison of enzalutamide and bicalutamide, only enzalutamide resulted in a significant reduction of tumor volume. No antitumor activity was observed with RD162 in castrate DU145 xenografts (an AR-deficient model), suggesting the critical role of persistent AR expression. Given the compelling evidence for clinical activity in relevant castrate-resistant models, clinical studies of these compounds were then undertaken. 


\subsection{Phase I/II Assessment}

Enzalutamide was assessed in a phase I/II study that included a total of 140 patients with CRPC. Patients included in the study were required to have a testosterone level $<50 \mathrm{ng} / \mathrm{dL}$ in association with a rising PSA. The presence of metastatic disease was not a requirement for the study. Although the study was initially conducted with a conventional $3+3$ design in the dose-escalation phase, PSA declines were noted in all 6 patients treated at a dose of 30 $\mathrm{mg}$ daily. Given this signal of efficacy, the study was expanded to explore larger cohorts at each level. These cohorts were comprised of distinct populations based on dose - between doses of 60 and $360 \mathrm{mg}$ daily, a total of 12 chemotherapy-naïve and 12 chemotherapyrefractory patients were enrolled at each level. In contrast, at the highest dose levels explored (480 and $600 \mathrm{mg}$ ), only patients with prior chemotherapy exposure were included. Prostate Cancer Working Group 2 (PCWG2) criteria were utilized to evaluate clinical outcome, and Response Evaluation Criteria in Solid Tumors (RECIST) parameters were used to characterize response in soft tissue disease. Although PCWG2 criteria offer a formal definition of PSA progression, the study protocol stipulated that patients should be maintained on therapy until the time of documented radiographic progression.

Pharmacokinetic studies accompanying the phase I component of the study identified a drug half-life of roughly one week, with a maximum serum concentration achieved between 30 minutes and 4 hours. A steady-state plateau was reached after roughly one month of therapy. Ultimately, the median time to PSA progression (TTPP) was 32 weeks for the overall cohort. TTPP was slightly prolonged in those patients who were chemotherapy-naïve as opposed to those patients with prior chemotherapy exposure (32 weeks $v 21$ weeks). Median time to radiographic progression (TTRP) was 47 weeks in the overall cohort, and again slightly higher in those patients who were chemotherapy-naïve (not reached, NR, v 29 weeks); $\mathrm{P}=0.01$ ). Fatigue was the most commonly observed adverse event, with dose reduction required amongst patients treated at $240 \mathrm{mg}$ or greater. A concerning signal for seizure emerged at higher doses of enzalutamide. Specifically, two patients had witnessed seizures at doses of 360 and $600 \mathrm{mg}$ daily, while a third patient treated at $480 \mathrm{mg}$ daily had a possible seizure. Myocardial infarction and rash represented other significant adverse events leading to treatment discontinuation. The frequency of adverse events that led to treatment discontinuation was higher in those patients treated at doses of $360 \mathrm{mg}$ daily or greater as compared to those treated at $240 \mathrm{mg}$ daily or less (13\% vs $1 \%$, respectively). Given this observation, along with the seizure events noted at doses at or above $360 \mathrm{mg}$ daily, $240 \mathrm{mg}$ daily was determined to be the maximally tolerated dose (MTD).

Several correlative studies accompanied this phase I/II effort. 22 patients treated at Memorial Sloan-Kettering Cancer Center treated on the parent protocol consented to further assessment with ${ }^{18} \mathrm{~F}$-dihydrotestosterone (DHT) PET. Patients were assessed at baseline and after 4 weeks of therapy, and the majority of patients had a $50 \%$ or greater reduction in maximal standard uptake value (SUV). Circulating tumor cells (CTCs) were collected for the majority of patients enrolled (128 of 140 patients, or $91 \%$ ). In accordance with previously published data, those patients with baseline CTC counts of $>5$ cells $/ 7.5 \mathrm{~mL}$ were characterized as unfavorable. ${ }^{17}$ Of the 51 patients with unfavorable counts, 25 patients (49\%) had a decline to $<5 \mathrm{CTCs} / 7.5 \mathrm{~mL}$.

\subsection{Phase III Assessment}

To date, one phase III assessment of enzalutamide has been published. Scher et al reported a comparison of enzalutamide and placebo in 1,199 patients with mCRPC in the AFFIRM study. ${ }^{8}$ Eligibility criteria for the study included castration-resistant disease (testosterone < $50 \mathrm{ng} / \mathrm{dL}$ ) with either PSA or radiographic progression according to PCWG2 criteria. 
Patients must also have had prior treatment with docetaxel. Stratification factors incorporated in the randomization included ECOG performance status and scores derived from the Brief Pain Inventory-Short Form (BPI-SF).

Patients were randomized in a 2:1 fashion to enzalutamide at $160 \mathrm{mg}$ daily vs. placebo. ${ }^{8}$ The rationale for dosing at $160 \mathrm{mg}$ daily was unspecified - an interesting selection given that the phase I/II study identified an MTD of $240 \mathrm{mg}$ daily. The primary endpoint for the study was overall survival (OS), with secondary endpoints including progression and response (based on PSA, radiographic evaluation and quality of life metrics).

Patients remained on enzalutamide for a median of 8.3 months, as compared to 3.0 months on placebo. ${ }^{8}$ The study achieved its primary endpoint, demonstrating an improvement in survival with enzalutamide (18.4 months $v 13.6$ months). In a multivariate analysis including study treatment, ECOG score, pain score, progression at study entry (PSA only $v$ radiographic), presence or absence of visceral disease, and baseline hemoglogin/LDH, treatment with enzalutamide was a predictor of OS. The study demonstrated improvement across all of the secondary endpoints. Both TTPP and TTRP tracked with the duration of study therapy and were improved with enzalutamide. TTPP was 8.3 months with enzalutamide as compared to 3.0 months with placebo $(\mathrm{P}<0.001)$, while TTRP was 8.3 months with enzalutamide as compared to 2.9 months with placebo $(\mathrm{P}<0.001)$. Quality of life as assessed by the Functional Assessment of Cancer Therapy-Prostate (FACT-P) scale was also significantly improved with enzalutamide $(\mathrm{P}<0.001)$.

Interestingly, the rate of grade $3 / 4$ adverse events with enzalutamide was lower than the rate with placebo $(45.3 \% v 53.1 \%) .{ }^{8}$ The most common adverse events associated with enzalutamide included fatigue, diarrhea and hot flashes. Again, a concerning safety signal emerged in the phase III trial - 5 patients on the enzalutamide arm had reported seizures, while no seizures were encountered in the placebo arm. Although the eligibility criteria for the AFFIRM study included strict prohibition of any agents that reduced the seizure threshold (i.e., droperidol, lidocaine, buproprion, insulin, lithium, etc.) or enrollment of patients with risk factors for seizure (i.e., prior seizure, syncopal episodes, or head trauma), one patient had received lidocaine inadvertently prior to experiencing a seizure. Two patients had documented brain metastases, and another had alcohol intoxication in association with the seizure.

\subsection{Expert Opinion}

The data from the AFFIRM trial led to approval of the agent on August 31, 2012 for patients with $\mathrm{mCRPC}$ who had received docetaxel. ${ }^{18}$ As noted, several other agents have also been approved for $\mathrm{mCPRC}$ over the past five years, including abiraterone, sipuleucel-T, and cabazitaxel. Other agents are also being evaluated, including ARN-509 and radium-223. ${ }^{19-21}$ ARN-509 (a novel antiandrogen) demonstrated activity in a phase I/II trial and may soon enter phase III testing, while radium-223 demonstrated a survival advantage in the phase III ALSYMPCA study, which may result in FDA approval in the near future. ${ }^{19-22}$ Prior to 2009, docetaxel was the only agent that had demonstrated a survival benefit in the setting of castration resistant disease. Now multiple therapeutic options are available.

Currently, there is a logical sequence in which to utilize post-docetaxel therapies.

Enzalutamide, abiraterone, and cabazitaxel produce a survival advantage in this setting, although abiraterone will now be used in the pre-docetaxel space given data from the COUAA-302 study and subsequent FDA approval. ${ }^{6}$ Comparing the degree of benefit associated with enzalutamide and cabazitaxel is challenging because of the difference in comparators in the pivotal studies (enzalutamide was compared to placebo, while cabazitaxel was compared 
to mitoxantrone). Nonetheless, the toxicity profile of enzalutamide appears to be far more modest than that of cabazitaxel, which led to appreciable rates of diarrhea and febrile neutropenia. It therefore seems that the practitioner is more likely to sequence enzalutamide prior to cabazitaxel. There may be rare instances when cytotoxic therapy is favored, such as in the setting of neuroendocrine disease (representing approximately $4 \%$ of all prostate cancer diagnoses). ${ }^{23}$ However, the AFFIRM trial excluded patients on drugs lowering the seizure threshold. This rather expansive list includes a number of commonly prescribed cardiovascular, neurologic and psychiatric agents. Given that patients with mCRPC are typically older and may have significant comorbidities, this could limit the real-world application of enzalutamide.

The clinical application of enzalutamide may change substantially with the upcoming results from the PREVAIL study (Figure 2). This study randomized mCRPC patients who had not been exposed to docetaxel to either enzalutamide or placebo. A press release on June 6, 2012, announced completion of enrollment of 1,680 patients. ${ }^{24}$ Similar to COU-AA-302, the prechemotherapy phase III assessment of abiraterone, the PREVAIL study includes coprimary endpoints of progression-free survival (PFS) and OS. If the PREVAIL study is positive, it will be an interesting challenge to appropriately sequence therapies for chemotherapy-naïve disease especially in the absence of level 1 evidence. There are already questions asking whether sipuleucel-T or abiraterone/prednisone should be used first in this disease state. If abiraterone and enzalutamide have similar clinical benefit and safety in their respective phase III assessments, enzalutamide may be preferred due to the lack of requirement for concomitant steroids. Emerging retrospective studies have assessed the efficacy of abiraterone subsequent to enzalutamide (to the author's knowledge, the opposite sequence has not been characterized). ${ }^{25-27}$ Noonan et al reported a small study in 25 patients with mCRPC from 3 Canadian centers. ${ }^{26}$ All had received abiraterone after prior enzalutamide and docetaxel. In 21 evaluable patients, only 3 patients (14\%) had a $>30 \%$ PSA decline with abiraterone - a lesser response rate than anticipated in the post-docetaxel setting. A similar population was assessed by Bianchini et al. ${ }^{27}$ In a series of 39 patients with prior docetaxel and abiraterone exposure, enzalutamide resulted in a $>30 \%$ PSA decline in only 6 patients $(15 \%)$. While larger studies are needed, these estimates suggest that the sequencing of enzalutamide and abiraterone may be an important decision, given the potential for cross-resistance.

Rational combinations of novel agents for mCRPC may ultimately represent the optimal strategy. For example, a phase II study is currently underway in which abiraterone and enzalutamide are administered simultaneously to patients with CRPC metastatic to bone. ${ }^{28}$ The primary endpoint of the study is to assess the safety and tolerability of the combination. In the future, a larger study that compares the combination of drugs to either sequence of drugs (i.e., abiraterone followed by enzalutamide or vice versa) could definitively identify the best practice for the oncology clinic. However, such a trial would require thousands of patients who would be lost to other studies.

Even if combinations were proven to be superior to sequential therapy, cost could be a problem. In fact, with the challenging fiscal climate facing the most modern healthcare system, cost may even serve as a driving force in the sequencing of monotherapies. At $\$ 7,450$ per month, enzalutamide will cost an average of $\$ 59,600$ per patient (assuming a median of 8 cycles is administered). This cost is expected to rise if enzalutamide moves into the prechemotherapy space, as a greater number of cycles will likely be given. The experience with abiraterone supports this idea - in the post-docetaxel COU-AA-301 trial, abiraterone was administered for a median of 8 months, while in the pre-docetaxel COUAA-302 study, patients received a median of 14 months of therapy. ${ }^{29}$ Assuming a monthly cost of $\$ 5,000$ for abiraterone, drug costs may thus increase by $\$ 30,000$ with transition of 
the abiraterone into the pre-chemotherapy space. As noted in Figure 2, if a patient were able to tolerate each of the approved agents for mCPRC, the drug cost would amount to $\$ 294,350$ per patient on average (Figure 3).

This suggests that pharmacoeconomics will play a key role in dictating therapy in the coming years. However, if every patient with mCRPC cannot access the full spectrum of therapies, then which ones will be offered? Here, it may be helpful to consider relevant biomarkers that can discern appropriate candidates for therapy. Mutated AR may represent one potential biomarker - however, the frequency of mutation is uncertain, with studies suggesting a frequency anywhere between $10-100 \% .{ }^{30-31}$ Certain mutations may mitigate the efficacy of therapeutic agents. For example, Li et al have demonstrated that AR splice variants that lack the androgen LBD confer resistance to enzalutamide. ${ }^{15}$ Although still requiring clinical validation, patients bearing such mutations may be more suitable candidates for cytotoxic therapy. In contrast, patients with amplification of the native AR with an intact LBD may derive more substantial benefit from enzalutamide. The frequency of AR amplification has also been reported with varying frequency, largely depending on the assay utilized. Using the Affymetrix SNP platform, one study identified AR amplification in 13 of 14 patients (93\%) in a small study. ${ }^{32}$ However, the use of FISH in the same cohort only identified 5 patients (36\%) with AR amplification. Clearly, these assays must be refined prior to further clinical implementation.

Several barriers exist to the use of biomarkers in mCRPC therapy. First, accessing tumor tissue in patients with $\mathrm{mCRPC}$ is challenging. Efstathiou et al recently reported a study in which 57 patients with mCRPC to bone received trans-iliac bone-marrow biopsies accompanying therapy with abiraterone. ${ }^{33}$ Of 57 patients enrolled, tumor-infiltrated bone marrow samples were obtained in only 25 patients (44\%). Given the challenges in obtaining metastatic tissue, attention has turned to individual analysis of CTCs. FISH assessment for AR or TMPRSS 2 mRNA is now feasible in single cells, and mRNA levels for each can be quantified. ${ }^{17}$ However, the studies to date that have explored these assays suggest high intrapatient variability. A second barrier to the use of biomarkers in mCRPC is the size of the clinical trials that are needed for validation. Kohli et al provide a detailed account of three relevant trial designs which employ molecular stratification. ${ }^{30}$ Each design begins with a heterogeneous population of patients with $\mathrm{mCRPC}$, and ultimately randomizes a subset of these patients based on the presence or absence of a biomarker. Previous estimates suggest that such clinical trials would require thousands of patients and it is unclear whether sufficient motivation exists within the biotech or pharmaceutical industries to support such efforts. ${ }^{34}$

It is now clear that patients with mCRPC are no longer devoid of options. Enzalutamide is the fourth agent to gain FDA approval since 2009, and dependent upon the results of the PREVAIL trial, it is possible that its use may shift into the pre-chemotherapy space. Beyond efficacy and safety, pharmacoeconomic considerations may also increasingly guide the sequencing of enzalutamide and other treatments for mCRPC. Given that the cumulative cost of drugs for the patient with mCRPC may approach $\$ 300,000$ per patient, difficult decisions regarding the appropriate extent of therapy will need to be made. In the future, molecular testing may be used to identify the most ideal candidates for enzalutamide therapy if sufficient enthusiasm and financial support can be generated for prospective biomarkerbased studies.

\section{Acknowledgments}

This work was supported by NIH K12 2K12CA001727-16A1 (S.K.P.). 


\section{REFERENCES}

1. Kantoff PW, Higano CS, Shore ND, et al. Sipuleucel-T Immunotherapy for Castration-Resistant Prostate Cancer. New England Journal of Medicine. 2010; 363(5):411-22. [PubMed: 20818862]

2. [last accessed January 6, 2013] FDA Approval Letter for Provenge (Sipuleucel-T). Available at http://www.fda.gov/BiologicsBloodVaccines/CellularGeneTherapyProducts/ApprovedProducts/u $\mathrm{cm} 210215 . \mathrm{htm}$

3. de Bono JS, Oudard S, Ozguroglu M, et al. Prednisone plus cabazitaxel or mitoxantrone for metastatic castration-resistant prostate cancer progressing after docetaxel treatment: a randomised open-label trial. The Lancet. Oct 2; 2010 376(9747):1147-54.

4. [last accessed March 18, 2012] FDA Approval Information for Cabazitaxel. Available at http:// www.cancer.gov/cancertopics/druginfo/fda-cabazitaxel

5. de Bono JS, Logothetis CJ, Molina A, et al. Abiraterone and increased survival in metastatic prostate cancer. N Engl J Med. May 26; 2011 364(21):1995-2005. [PubMed: 21612468]

6. Ryan CJ, Smith MR, de Bono JS, et al. Abiraterone in Metastatic Prostate Cancer without Previous Chemotherapy. N Engl J Med. Dec 10.2012

7. [last accessed January 6, 2012] FDA Approval for Abiraterone Acetate. Available at http:// www.cancer.gov/cancertopics/druginfo/fda-abirateroneacetate

8. Scher HI, Fizazi K, Saad F, et al. Increased survival with enzalutamide in prostate cancer after chemotherapy. N Engl J Med. Sep 27; 2012 367(13):1187-97. [PubMed: 22894553]

9. [last accessed January 6, 2013] FDA Approval for Enzalutamide. Available at http:// www.cancer.gov/cancertopics/druginfo/fda-enzalutamide

10. Taplin ME, Rajeshkumar B, Halabi S, et al. Androgen receptor mutations in androgen-independent prostate cancer: Cancer and Leukemia Group B Study 9663. J Clin Oncol. Jul 15; 2003 21(14): 2673-8. [PubMed: 12860943]

11. Matias PM, Carrondo MA, Coelho R, et al. Structural basis for the glucocorticoid response in a mutant human androgen receptor (AR(ccr)) derived from an androgen-independent prostate cancer. J Med Chem. Mar 28; 2002 45(7):1439-46. [PubMed: 11906285]

12. Veldscholte J, Ris-Stalpers C, Kuiper GG, et al. A mutation in the ligand binding domain of the androgen receptor of human $\mathrm{LNCaP}$ cells affects steroid binding characteristics and response to anti-androgens. Biochem Biophys Res Commun. Dec 14; 1990 173(2):534-40. [PubMed: 2260966]

13. Craft N, Shostak Y, Carey M, et al. A mechanism for hormone-independent prostate cancer through modulation of androgen receptor signaling by the HER-2/neu tyrosine kinase. Nat Med. Mar; 1999 5(3):280-5. [PubMed: 10086382]

14. Chen CD, Welsbie DS, Tran C, et al. Molecular determinants of resistance to antiandrogen therapy. Nat Med. Jan; 2004 10(1):33-9. [PubMed: 14702632]

15. Li Y, Chan SC, Brand LJ, et al. Androgen receptor splice variants mediate enzalutamide resistance in castration-resistant prostate cancer cell lines. Cancer Res. Nov 1.2012

16. Tran C, Ouk S, Clegg NJ, et al. Development of a Second-Generation Antiandrogen for Treatment of Advanced Prostate Cancer. Science. May 8; 2009 324(5928):787-90. 2009. [PubMed: 19359544]

17. Danila DC, Fleisher M, Scher HI. Circulating Tumor Cells as Biomarkers in Prostate Cancer. Clinical Cancer Research. 2011; 17(12):3903-12. [PubMed: 21680546]

18. [last accessed January 3, 2012] FDA Approval Letter for Enzalutamide. Available at http:// www.fda.gov/Drugs/InformationOnDrugs/ApprovedDrugs/ucm317997.htm

19. Clegg NJ, Wongvipat J, Joseph JD, et al. ARN-509: a novel antiandrogen for prostate cancer treatment. Cancer Res. Mar 15; 2012 72(6):1494-503. [PubMed: 22266222]

20. Parker C, Heinrich D, O'Sullivan JM, et al. Overall survival benefit and safety profile of radium-223 chloride, a first-in-class alpha-pharmaceutical: Results from a phase III randomized trial (ALSYMPCA) in patients with castration-resistant prostate cancer (CRPC) with bone metastases. ASCO Meeting Abstracts. 2012; 30(5_suppl):8. 
21. Sartor AO, Heinrich D, Helle SI, et al. Radium-223 chloride impact on skeletal-related events in patients with castration-resistant prostate cancer (CRPC) with bone metastases: A phase III randomized trial (ALSYMPCA). ASCO Meeting Abstracts. 2012; 30(5_suppl):9.

22. Smith, MR.; Antonarakis, ES.; Ryan, CJ., et al. ARN-509 in men with high-risk non metastatic castration resistant prostate cancer Presented at the 2012 European Society of Medical Oncology Meeting in Vienna; Austria. [Abstr 3109]

23. Culine S, El Demery M, Lamy P-J, et al. Docetaxel and Cisplatin in Patients With Metastatic Androgen Independent Prostate Cancer and Circulating Neuroendocrine Markers. The Journal of urology. 2007; 178(3):844-48. [PubMed: 17631339]

24. [last accessed January 4, 2013] Medivation Press Release: PREVAIL Enrollment. Available at http://investors.medivation.com/releasedetail.cfm?ReleaseID=680579

25. Ileana E, Loriot Y, Albiges L, et al. Abiraterone in patients with metastatic castration-resistant prostate cancer progressing after docetaxel and MDV3100. ASCO Meeting Abstracts. 2012; 30(15_suppl):4554.

26. Noonan, K.; Ellard, S.; Chi, K. Clinical activity of abiraterone acetate (AA) after progression on MDV3100 in patients with metastatic castration resistant prostate cancer (mCRPC). Presented at the 2012 European Society of Medical Oncology Meeting in Vienna; Austria. [Abstr 925P]

27. Bianchini, D.; Loriot, Y.; Ileana, E., et al. Abiraterone in patients with metastatic castration resistant prostate cancer progressing after docetaxel and MDV3100: A multicentre study. Presented at the 2012 European Society of Medical Oncology Meeting in Vienna; Austria. [Abstr 924P]

28. [last accessed January 4, 2013] NCT01650194: A Phase 2 Study Determining Safety and Tolerability of Enzalutamide (Formerly MDV3100) in Combination With Abiraterone Acetate in Bone Metastatic Castration-Resistant Prostate Cancer Patients. Available at http:// clinicaltrials.gov/ct2/show/NCT01650194

29. Basch, E.; Ryan, CJ.; Kheoh, T., et al. The impact of abiraterone acetate (AA) therapy on patientreported pain and functional status in chemotherapy-naive patients with progression metastatic castration-resistant prostate cancer. Presented at the 2012 European Society of Medical Oncology Meeting in Vienna; Austria. 2012. [Abstr 8950]

30. Kohli M, Qin R, Jimenez R, et al. Biomarker-based targeting of the androgen-androgen receptor axis in advanced prostate cancer. Adv Urol. 2012; 2012:781459. [PubMed: 22956944]

31. Taplin ME, Bubley GJ, Shuster TD, et al. Mutation of the androgen-receptor gene in metastatic androgen-independent prostate cancer. N Engl J Med. May 25; 1995 332(21):1393-8. [PubMed: 7723794]

32. Liu W, Laitinen S, Khan S, et al. Copy number analysis indicates monoclonal origin of lethal metastatic prostate cancer. Nat Med. May; 2009 15(5):559-65. [PubMed: 19363497]

33. Efstathiou E, Titus M, Tsavachidou D, et al. Effects of abiraterone acetate on androgen signaling in castrate-resistant prostate cancer in bone. J Clin Oncol. Feb 20; 2012 30(6):637-43. [PubMed: 22184395]

34. Sargent DJ, Conley BA, Allegra C, et al. Clinical Trial Designs for Predictive Marker Validation in Cancer Treatment Trials. Journal of Clinical Oncology. Mar 20; 2005 23(9):2020-27. 2005.

[PubMed: 15774793] 


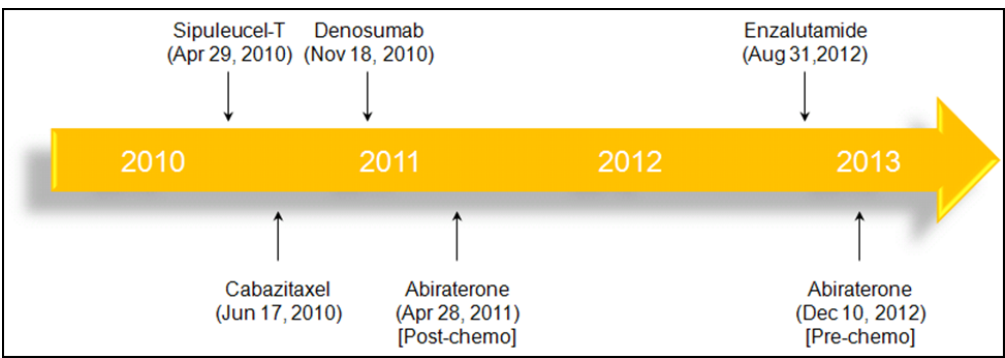

Figure 1.

Key approvals for mCRPC over the past 3 years. 


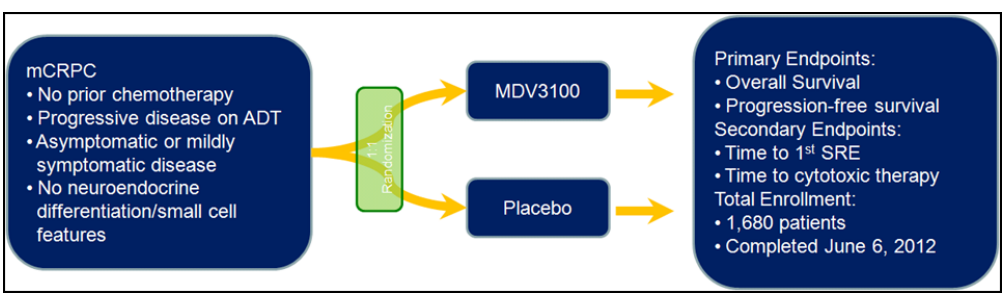

Figure 2.

Schema for the recently completed phase III PREVAIL study. 


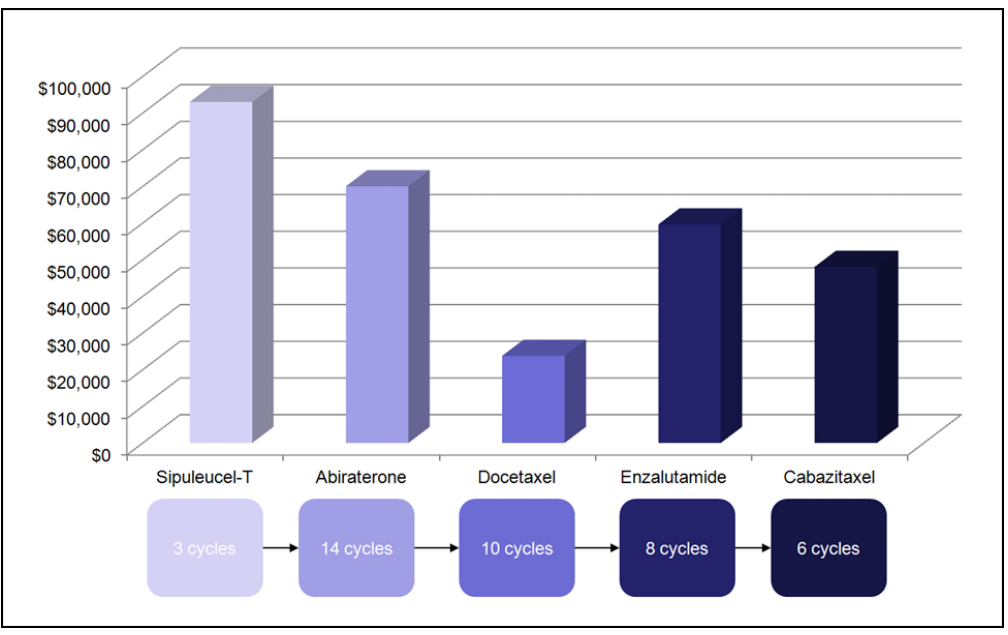

Figure 3.

Average costs associated with a standard sequence of therapies for mCRPC. Assuming that a patient receives the median number of cycles reported in pivotal phase III studies, the total drug cost amounts to $\$ 294,350$. 
Table 1

Drug summary.

\begin{tabular}{|l|l|}
\hline Drug Name & Enzalutamide \\
\hline Phase & III \\
\hline Indication & Metastatic castration-resistant prostate cancer \\
\hline Pharmacology & Antiandrogen \\
\hline Route of Administration & Oral \\
\hline & \\
Chemical Structure & AFFIRM, PREVAIL \\
\hline Pivotal Trials &
\end{tabular}

